\title{
Dynamical modes associated with the Antarctic ozone hole
}

\author{
B. C. Weare \\ Atmospheric Science Program, Department of Land, Air and Water Resources, University of California-Davis, Davis, CA \\ 95616, USA
}

Received: 11 December 2008 - Published in Atmos. Chem. Phys. Discuss.: 25 February 2009

Revised: 9 July 2009 - Accepted: 13 July 2009 - Published: 3 August 2009

\begin{abstract}
Generalized Maximum Covariance Analysis (GMCA) has been developed and applied to diagnosing the dynamical modes associated with variations in the Antarctic spring ozone hole. GMCA is used to identify the most important patterns of co-variability between interannual ozone mixing ratio variations in the Antarctic region and temperature, zonal, meridional and vertical velocities between 100 and $10 \mathrm{hPa}$ in the same region. The most important two pairs of GMCA time coefficients show large year-to-year variations and trends, which are connected with the growth of the Antarctic Ozone Hole and the increase of ozone depleting substances. The associated spatial patterns of ozone variations may be characterized as being quasi-symmetric and asymmetric about the pole. These patterns of ozone variations are associated with comparable patterns of variations of temperature and winds through most of the vertical domain.

The year 2000 is shown to be dominated by the asymmetric mode, whereas the adjacent year 2001 is dominated by the quasi-symmetric mode. A case study, focusing on the asymmetric differences between these two years, shows the magnitude of the ozone mixing ratio, temperature and zonal wind differences to be in the range of $2 \mathrm{e}-6 \mathrm{~kg} / \mathrm{kg}, 10^{\circ} \mathrm{C}$ and $10 \mathrm{~m} / \mathrm{s}$, respectively. Budget calculations show that transport processes contribute substantially to the ozone and temperature changes in the middle stratosphere over the Antarctic continent. However, both radiative and chemical processes also play important roles in the changes.
\end{abstract}

\section{Introduction}

The story of the Antarctic ozone hole illustrates perhaps the greatest disaster and success of science in the twentieth century. During the late 1920s CFC's were developed as the

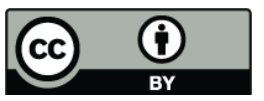

Correspondence to: B. C. Weare (bcweare@ucdavis.edu) ideal fluid for refrigerants, combining effective thermodynamic properties in a safe material, which was thought to be essentially inert. In the mid 1970s it came to be understood that these and related materials are dissociated by ultraviolet radiation in the stratosphere and the released chlorine leads to ozone destruction. In the early 1980s it was recognized that this destruction was far more effective near Antarctica in the spring leading to what we now call the ozone hole. The culmination of these rapid scientific advances was the series of international regulatory agreements, generally labeled the Montreal Protocol, which led to the near total ban of the production of the related man-made ozone depleting chemicals.

The basics of the science of the ozone hole are summarized by Rowland (2006). Figure 1, using data from the ERA-40 reanalysis (Uppala et al., 2005), helps to illustrate some important aspects of these concepts. Rapid destruction of ozone in the early spring is associated with polar stratospheric clouds accompanying very cold temperatures near the South Pole, which are associated with a strong polar vortex. Between 2001 and 1979 there was a dramatic reduction in the ozone mixing ratio over much of the stratosphere due mainly to accelerated destruction due to increased concentrations of ozone depleting substances. In part this ozone loss led to a decrease in solar heating and a substantial decrease in temperature and increases in baroclinically driven zonal winds in this region. The decreased temperatures in turn may have led to more polar stratospheric clouds and more ozone depletion mediated by anthropogenic ozone depleting materials.

Scientists are now beginning to understand that ozone destruction in the recent past is being, and will continue to be, altered by the cooling of the stratosphere associated with "global warming". Furthermore, it is now better appreciated that the combined effects of ozone destroying chemicals and global warming are strongly tied to stratospheric dynamics and thermodynamics. This understanding is partially prompted by a recognition that many aspects of the ozone hole are not symmetric about the equator and that these

Published by Copernicus Publications on behalf of the European Geosciences Union. 


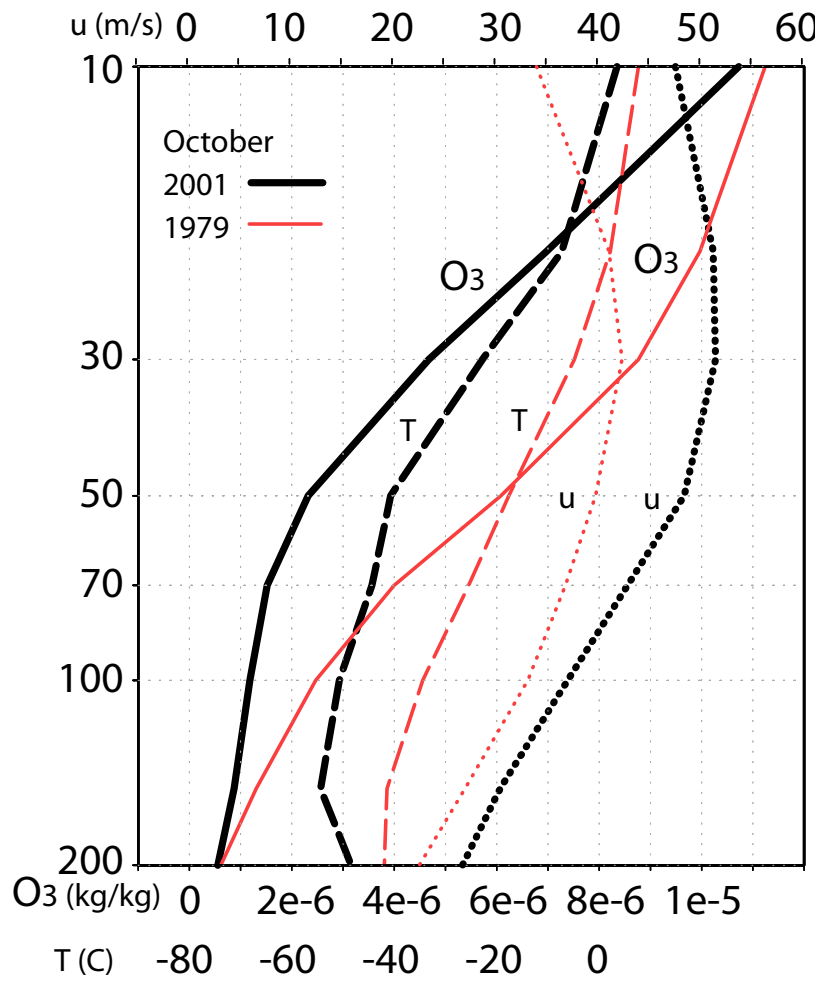

Fig. 1. Vertical profiles of the October 1979 (red) and 2001 (black). Ozone mixing ratio $\mathrm{O}_{3}$ (first lower scale) and temperature $T$ (second lower scale) south of $50^{\circ} \mathrm{S}$ and zonal wind $u$ (upper scale) between 45 and $55^{\circ} \mathrm{S}$. Vertical scale is in $\mathrm{hPa}$. Note that the 2001 values have lower $\mathrm{O}_{3}$ and $T$ and higher $u$ at all levels.

asymmetries differ substantially from year to year. For instance Grytsai et al. (2008) have pointed out that planetary waves over Antarctica played an especially important role in the ozone hole in 1988 and 2002.

Figure 2 illustrates this for October 1988 at the $50 \mathrm{hPa}$ level for ozone mixing ratio $\left(\mathrm{O}_{3}\right)$, temperature $(T)$, zonal $(u)$ and meridional $(v)$ winds and pressure velocity $(\omega)$, again taken from the ERA-40 reanalysis. Figure 2a shows an ozone hole which is shifted from the pole towards South America with an ozone maximum on the edge of the Antarctic continent south of Australia. These two sectors also correspond to a minimum and maximum in temperature. Accompanying these asymmetries is a shift in the Antarctic westerly wind vortex from the center of the continent towards South America, although the strongest westerlies are south of Africa. These shifts are associated with wave number one patterns of meridional and pressure velocities such that air at this level is flowing across the continent from the Pacific sector toward Africa with rising motion south of the Eastern Ocean and sinking south of Africa. In this case strong asymmetries are very evident even though for these plotted values neither zonal nor time means have been removed from the data.
The overall goal of this paper is to better understand the nature of the variations in the stratosphere accompanying ozone depletion near the Antarctic continent. A key question to be answered is how are year-to-year variations in the ozone hole related to variations in the dynamics of this region. To answer this and other questions a diagnosis of reanalysis data is performed for all of the Octobers between 1979 and 2001.

\section{Methods and data}

\section{$2.1 \quad$ ERA-40}

The European Centre for Medium-Range Weather Forecasting ERA-40 reanalysis (Uppala et al., 2005) includes output of many meteorological variables at standard levels to $1 \mathrm{hPa}$. These data are derived from a sophisticated assimilation of conventional and satellite data using a global weather forecast model. Although ERA-40 output is available starting in 1958, all-important satellite data only become fully available in 1978. Dethof and Hólm (2004) compare ERA40 ozone values with both land-based and satellite observations. They state that "ERA-40 ozone profiles also compare well with independent observations..." Dethof and Hólm also show that ERA-40 reproduces reasonably well ozone interannual variability at sample pressure levels. Uppala et al. (2005) and Randel et al. (2004) conclude that the ERA-40 analyses are generally skillful at representing the QBO and Northern Hemisphere Sudden Warming events; Le Sommer et al. (2006) come to a comparable conclusion concerning inertia-gravity wave activity. Overall, Uppala et al. (2005), Pascoe et al. (2005) and Randel et al. (2004) all conclude that the ERA-40 analyses are generally skillful at representing the most important aspects of stratospheric variations.

\subsection{Generalized Maximum Covariance Analysis (GMCA)}

Weare $(2008,2009)$ describes Generalized Maximum Covariance Analysis (MCA). In general this sophisticated diagnostic technique identifies the most important modes of covariability between two multi-dimensional data sets. The initial step in MCA is the calculation of a covariance (or correlation) matrix $\mathbf{C}$, derived from the products of time variations at each point in an $\mathbf{S} N_{s}$ point data set and those in a $\mathbf{Z}$ $N_{z}$ data set each having $M$ times.

$$
\begin{aligned}
& \mathbf{C}=\mathbf{S Z}^{T} / \mathbf{M} ; \mathbf{S}=\left(\begin{array}{cccc}
\mathbf{s}_{11} & \mathbf{s}_{12} & \bullet & \mathbf{s}_{1 M} \\
\mathbf{s}_{12} & \mathbf{s}_{22} & \bullet & \bullet \\
\bullet & \bullet & \bullet & \bullet \\
\mathbf{s}_{\mathbf{N} 1} & \bullet & \bullet & \mathbf{s}_{\mathbf{N}_{\mathrm{S}} \mathrm{M}}
\end{array}\right) \text {; } \\
& \mathrm{Z}=\left(\begin{array}{cccc}
\mathrm{z}_{11} & \mathrm{z}_{12} & \bullet & \mathrm{z}_{1 \mathrm{M}} \\
\mathrm{z}_{12} & \mathrm{z}_{22} & \bullet & \bullet \\
\bullet & \bullet & \bullet & \bullet \\
\mathrm{z}_{\mathrm{N} 1} & \bullet & \bullet & \mathrm{z}_{\mathrm{N}_{\mathrm{Z}} \mathrm{M}}
\end{array}\right)
\end{aligned}
$$




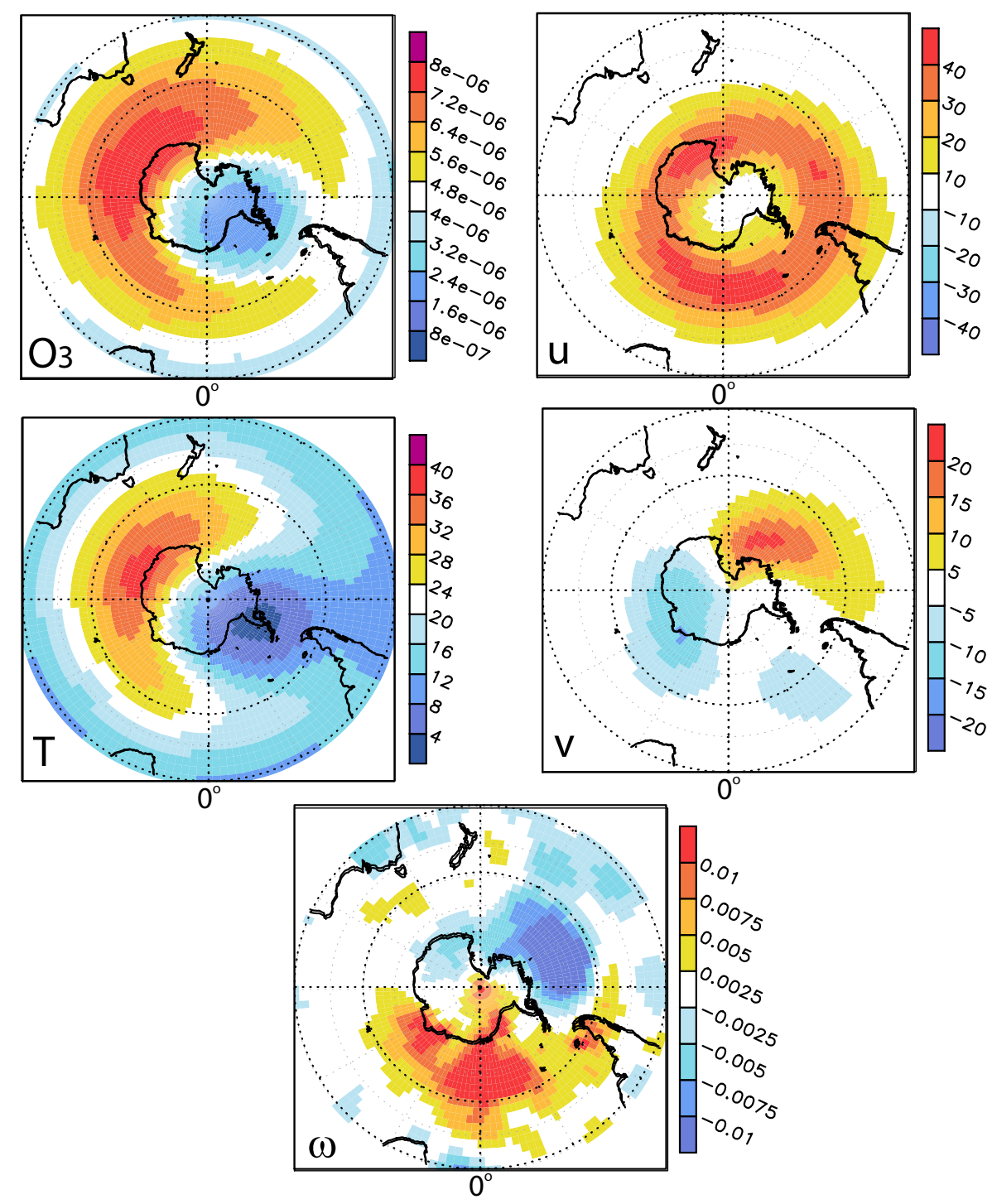

Fig. 2. October $198850 \mathrm{hPa}$ departures from the 1979-2001 October means: ozone mixing ratio $\mathrm{O}_{3}(\mathrm{~kg} / \mathrm{kg})$, temperature $T$ ( $\left.{ }^{\circ} \mathrm{C}\right)$, zonal wind $u(\mathrm{~m} / \mathrm{s})$, meridional wind $v(\mathrm{~m} / \mathrm{s})$, and pressure velocity $\omega(\mathrm{hPa} / \mathrm{s})$. The inner and outer concentric circles are $50^{\circ} \mathrm{S}$ and $30^{\circ} \mathrm{S}$. Note that the ozone hole and cold temperatures are largely confined to the South American sector.

This matrix is decomposed into right $\mathbf{R}$ and left $\mathbf{L}$ matrices of vectors associated with singular values $\omega_{i}$, such that

$$
\mathbf{C}=\mathbf{L} \omega \mathbf{R}^{T}
$$

where $\omega$ is an ordered diagonal matrix of singular values. The ordering is such that the first left $\mathbf{L}_{1}$ and right $\mathbf{R}_{1}$ vectors are associated with the largest explained squared covariance (correlation) between the $\mathbf{S}$ and $\mathbf{Z}$ fields. Thus, $\mathbf{L}_{i}$ and $\mathbf{R}_{i}$ for small i describe the dominant modes of co-variability of the input data $\mathbf{S}$ and $\mathbf{Z}$. Associated with these vectors, which are orthogonal in space, are the left $a_{i}(t)$ and right $b_{i}(t)$ time coefficients defined by inner products such that

$a_{i}(t)=\mathbf{L}_{i} \bullet \mathbf{S}(t) \quad ; \quad b_{i}(t)=\mathbf{R}_{i} \bullet \mathbf{Z}(t)$
The time series for the most important singular vectors (associated with the largest $\omega_{i}$ ) are the "best" representation of the time variations of corresponding data fields $\mathbf{S}$ and $\mathbf{Z}$. Temporal agreement between $a_{i}(t)$ and $b_{i}(t)$ is a measure of the co-variability of the mode i. Using these time coefficients one can define heterogeneous covariance maps, which are the products of the time series of the left vectors $a_{i}(t)$ with the right data $\mathbf{Z}$, and vice versa, such that

$$
\mathbf{r}_{S_{i}}=b_{i}(t) \bullet \mathbf{S}(t) ; \quad \mathbf{r}_{Z_{i}}=a_{i}(t) \bullet \mathbf{Z}(t)
$$

These heterogeneous covariance maps (or correlation maps for normalized data) describe the point-by-point agreement between the time coefficients associated with the variations of right singular vector $b_{i}(t)$ with the data field $\mathbf{S}$, and vice 

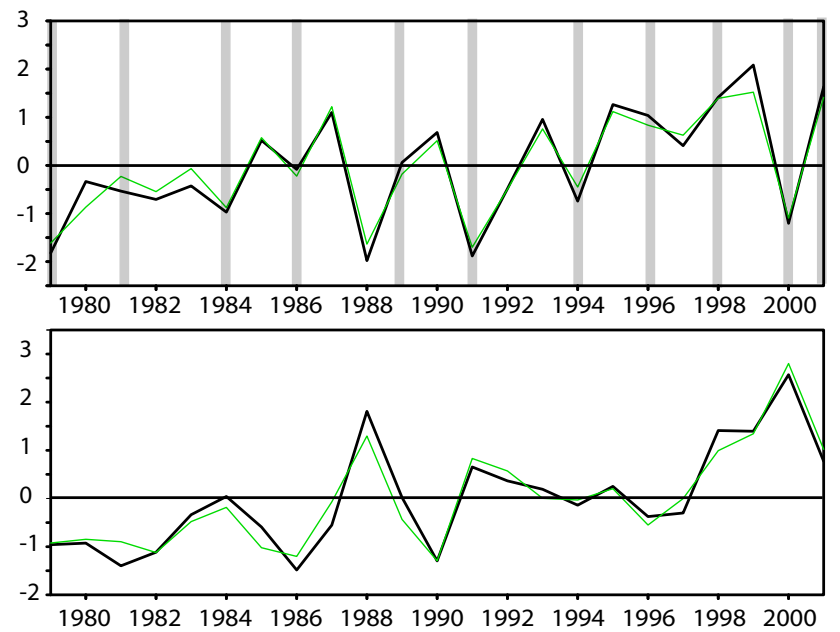

Fig. 3. GMCA 1 (top) and GMCA 2 (bottom) left $S(t)$ (heavy) and right $Z(t)$ October time coefficients.
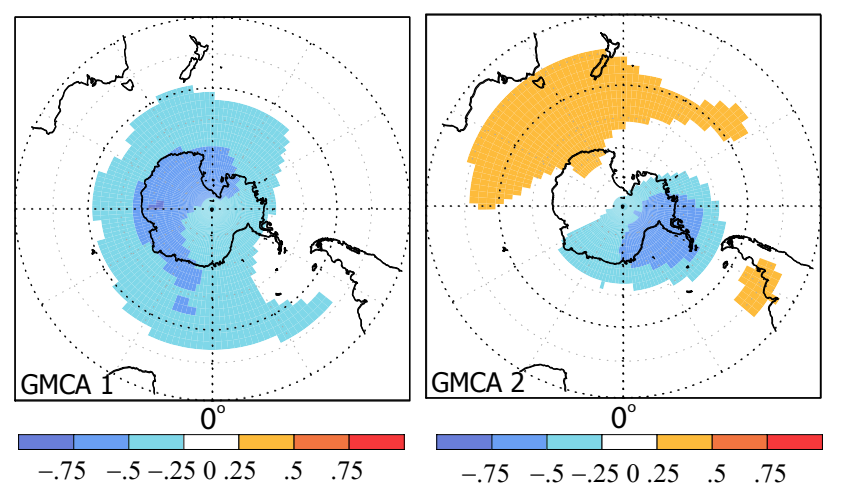

Fig. 4. Left heterogeneous correlations $\boldsymbol{r}_{S_{i}}$, corresponding to the GMCA1 and GMCA2 left vectors, representing the most important patterns of co-variability of $50 \mathrm{hPa}$ ozone mixing ratio with the $T$, $u, v$ and $\omega$ data fields. The inner and outer concentric circles are $50^{\circ} \mathrm{S}$ and $30^{\circ} \mathrm{S}$.

versa. For normalized input data these heterogeneous correlation maps have the same patterns as the corresponding left and right singular vectors (Bretherton et al., 1992).

The current version of Generalized MCA (GMCA; Weare, 2009) extends traditional MCA such that one of the analyzed variables (here designated as the $\mathbf{Z}$ set) is composed of multiple variables, levels and lags. Here the simpler $\mathbf{S}$ matrix is calculated from the first ten principle components (PC) of an Empirical Orthogonal Function (EOF) analysis of October ozone mixing ratio at $50 \mathrm{hPa}$ for the region south of $50^{\circ} \mathrm{S}$ for the years $1979-2001$. The $\mathbf{Z}$ matrix is calculated using the dominant PCs (normally 10) of EOF analyses of un-normalized departures from the long term monthly means of $T, u, v$, and $\omega$ (pressure velocity) for the region south of $30^{\circ} \mathrm{S}$ at six levels $(10,20,30,50,70$, and $100 \mathrm{hPa})$ for the months August, September, October, November and Decem-
Table 1. EOF explained variances for variables at the $50 \mathrm{hPa}$ level.

\begin{tabular}{cccc}
\hline Variable & $\begin{array}{c}\text { \% Variance } \\
\text { of EOF } 1\end{array}$ & $\begin{array}{c}\text { \% Variance first } \\
\text { 5 EOFs }\end{array}$ & $\begin{array}{c}\text { \% Variance first } \\
10 \text { EOFs }\end{array}$ \\
\hline $\mathrm{O}_{3}$ & 74 & 95 & 99 \\
$T$ & 52 & 91 & 99 \\
$u$ & 28 & 73 & 89 \\
$v$ & 26 & 77 & 92 \\
$\omega$ & 11 & 38 & 55 \\
\hline
\end{tabular}

Table 2. Generalized MCA statistics.

\begin{tabular}{ccc}
\hline GMCA & $\begin{array}{c}\text { \% squared correlation } \\
\text { explained }\end{array}$ & $\begin{array}{c}\text { Correlation between } \\
S(t) \text { and } Z(t)\end{array}$ \\
\hline 1 & 29.6 & 0.96 \\
2 & 24.0 & 0.97 \\
3 & 20.6 & 0.95 \\
4 & 17.8 & 0.95 \\
5 & 8 & 0.96 \\
\hline
\end{tabular}

ber for the years 1979-2001. To accommodate the very different units and magnitudes of both sets of input variables all PCs are normalized by their long term standard deviations. Most of the results will be displayed in terms of the heterogeneous correlations. Assuming that variations in separate years are independent, there are 23 degrees of freedom, and correlations with magnitudes greater than about 0.25 are significant at the 95\% confidence level (Von Storch and Zwiers, 1999).

\section{GMCA results}

As stated above the basic input data for the GMCA are the principal components of the most important Empirical Orthogonal Functions of the ozone, temperature, and wind data. Table 1 shows the variance explained by the first, the first five, and the first ten EOFs of these data. Clearly, the dominant ozone and temperature EOFs explain a large fraction of the variance; those of the zonal and meridional winds about a quarter of the variance; that of the pressure velocity about $10 \%$ of the variance. In all cases more than half of the variance is explained by the dominant ten EOFs with the smallest value again for the pressure velocity. In any case it is clear that 10 functions explain a large fraction of the non-noise variability.

Table 2 shows the percentage of the squared correlation explained by the first five GMCA functions and the correlations between the corresponding $a_{i}(t)$ and $b_{i}(t)$. What is evident in this table is that there is a great deal of correlation between the sets of variables so that the $a_{i}(t)$ and $b_{i}(t)$ 


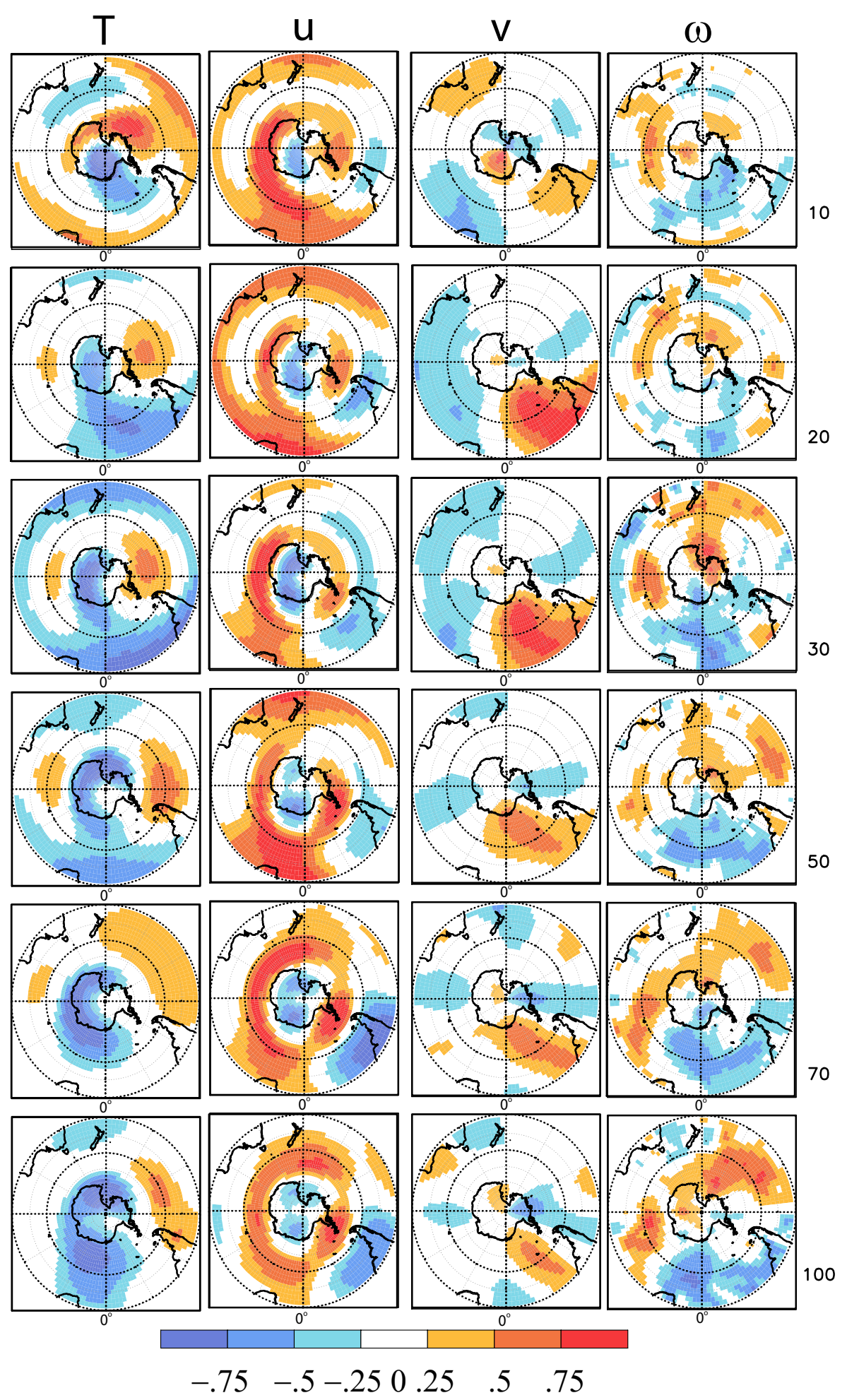

Fig. 5. Right heterogeneous correlations $\boldsymbol{r}_{Z_{i}}$ corresponding to the October GMCA1 right vectors at the pressure levels shown on the right $(\mathrm{hPa})$, representing the most important patterns of co-variability with $50 \mathrm{hPa}$ ozone mixing ratio. The inner and outer concentric circles are $50^{\circ} \mathrm{S}$ and $30^{\circ} \mathrm{S}$. 


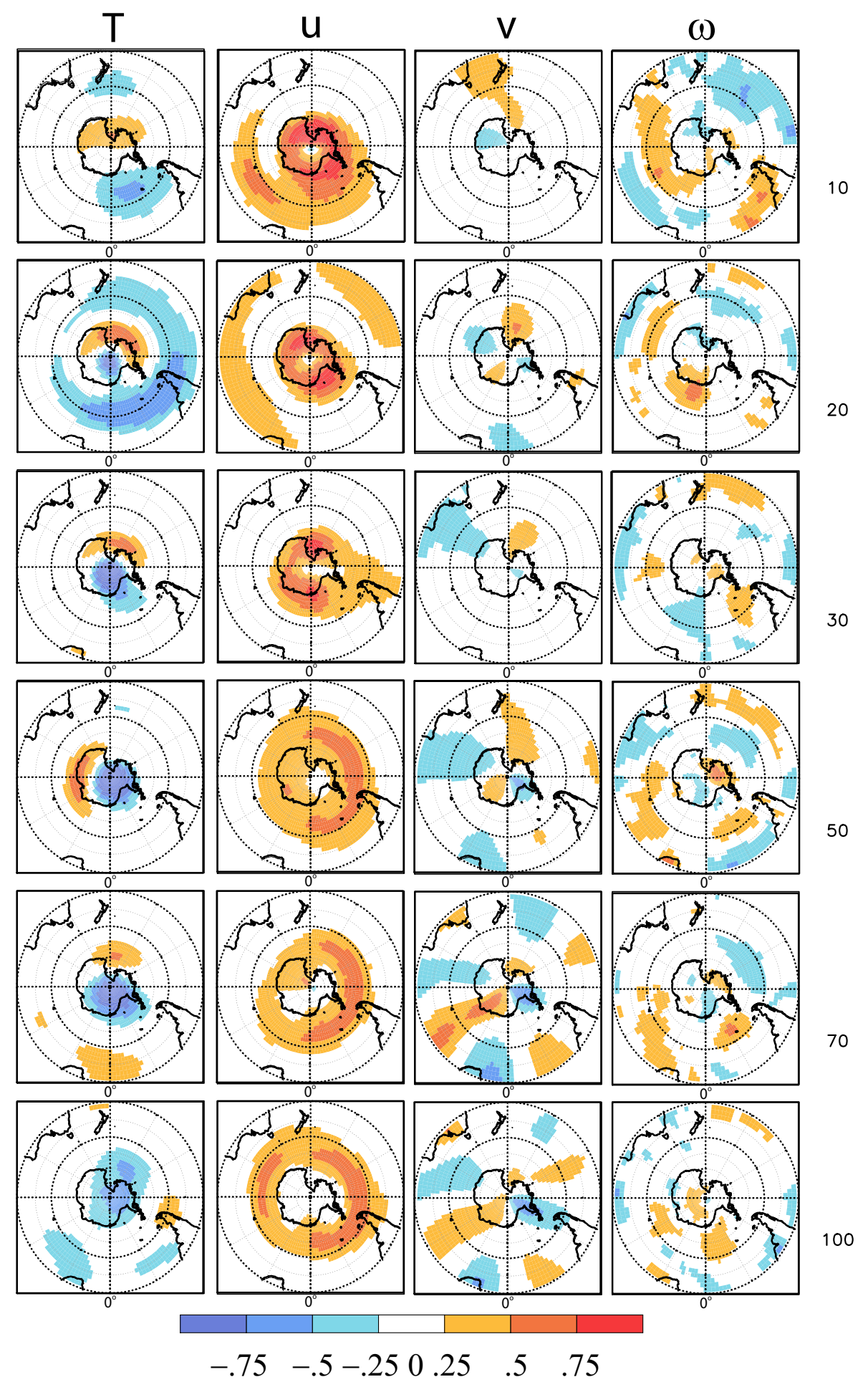

Fig. 6. Right heterogeneous correlations $\boldsymbol{r}_{Z_{11}}$ corresponding to the November GMCA1 right vectors at the pressure levels shown on the right (hPa). The inner and outer concentric circles are $50^{\circ} \mathrm{S}$ and $30^{\circ} \mathrm{S}$. 


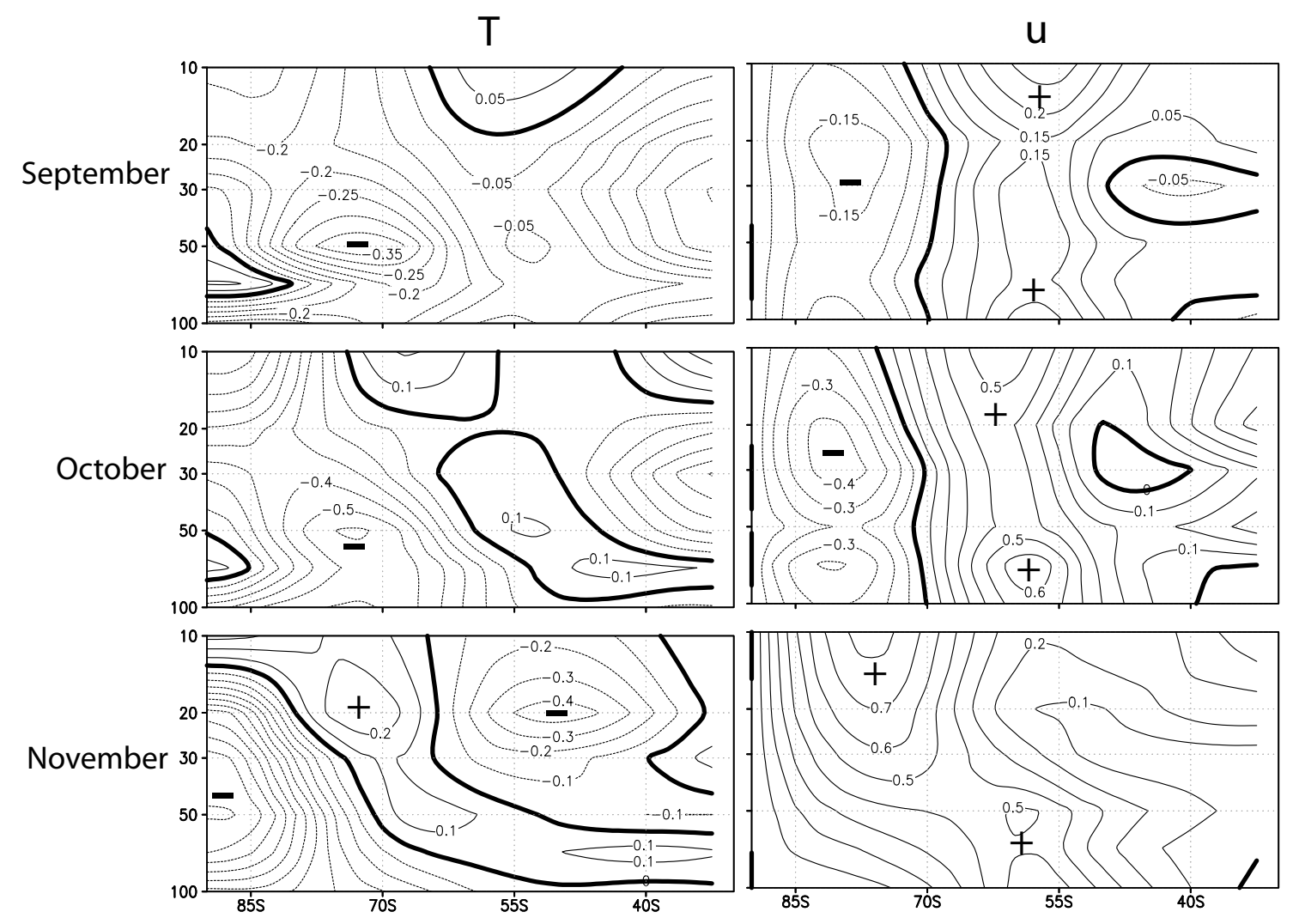

Fig. 7. Zonal averages of the right heterogeneous correlations $\boldsymbol{r}_{Z_{11}}$ for temperature (left) and zonal wind (right) for September, October and November at the pressure levels shown on the left $(\mathrm{hPa})$.

time series are very strongly correlated for all of the GMCAs. This is illustrated in Fig. 3, which shows the time coefficients for the first two GMCAs; both sets of coefficients have correlations greater than 0.95 . Also, both functions have moderately strong upward trends over the 23 years of the analysis. In addition to the trends there are relatively large variations, which have a quasi-period of $2-3$ years. Also, in Fig. 3 is a measure of the timing of negative phase of the tropical Quasi-Biennial Oscillation (QBO). Those Octobers in which the $30 \mathrm{hPa}$ zonal winds in the tropics are less than $-8 \mathrm{~m} / \mathrm{s}$ are indicated by vertical gray bars. There are suggestions that some, but by no means all, of the year-to-year variability in GMCA1 appears to be related to the QBO.

Figure 4 shows the $r_{S}$ (ozone departures) heterogeneous correlations patterns of the two most important GMCAs. $\mathbf{r}_{S_{1}}$ is negative over nearly the whole region and quasi-symmetric about the pole, whereas $\mathbf{r}_{S_{2}}$ shows the correlations of October ozone departures with the $b_{2}(t) s$, which are both positive and negative and have a pattern that is asymmetric about the pole. These plots identify two distinct modes of ozone variability. Together with the trends illustrated in Fig. 3 the broad negative regions in Fig. 4 illustrate the known downward trends in ozone and temperatures in the Antarctic region associated with the ozone hole.
As is evident from Table 3, GMCAs 3 and 4 explain almost as much of the covariability as do 1 and 2 . Their time coefficients (not shown) do not have distinct trends, and thus are not directly related to ozone depletion. However, the heterogeneous correlations $r_{S}$ also have quasi-symmetric and asymmetric spatial patterns. Because of the lack of trends in the time coefficients, emphasis in this paper will be on the first two GMCAs.

Figure 5 shows the $\mathbf{r}_{Z_{1}}$ correlation patterns for the first GMCA for October. Looking at temperature and zonal wind at all heights in the region of the Antarctic continent the correlations are primarily negative, and there is a strong suggestion of quasi-symmetry. At lower latitudes wave number one and two patterns are more evident. The meridional velocity correlation pattern suggests a flow into the pole in the Atlantic sector when the $a_{1}(t)$ coefficients are positive. The correlations of the pressure velocity show rising in the south Atlantic with the opposite motion across a broad expanse of the south Pacific. The temperature and zonal wind patterns are clearly identifiable with the quasi-symmetric ozone structure shown in Fig. 4.

Figure 6 shows the $\mathbf{r}_{Z_{1}}$ patterns for November. For $T$ and $u$ there is strong evidence of a tightening of the circumpolar vortex relative to that of October. Overall the $v$ and $\omega$ 


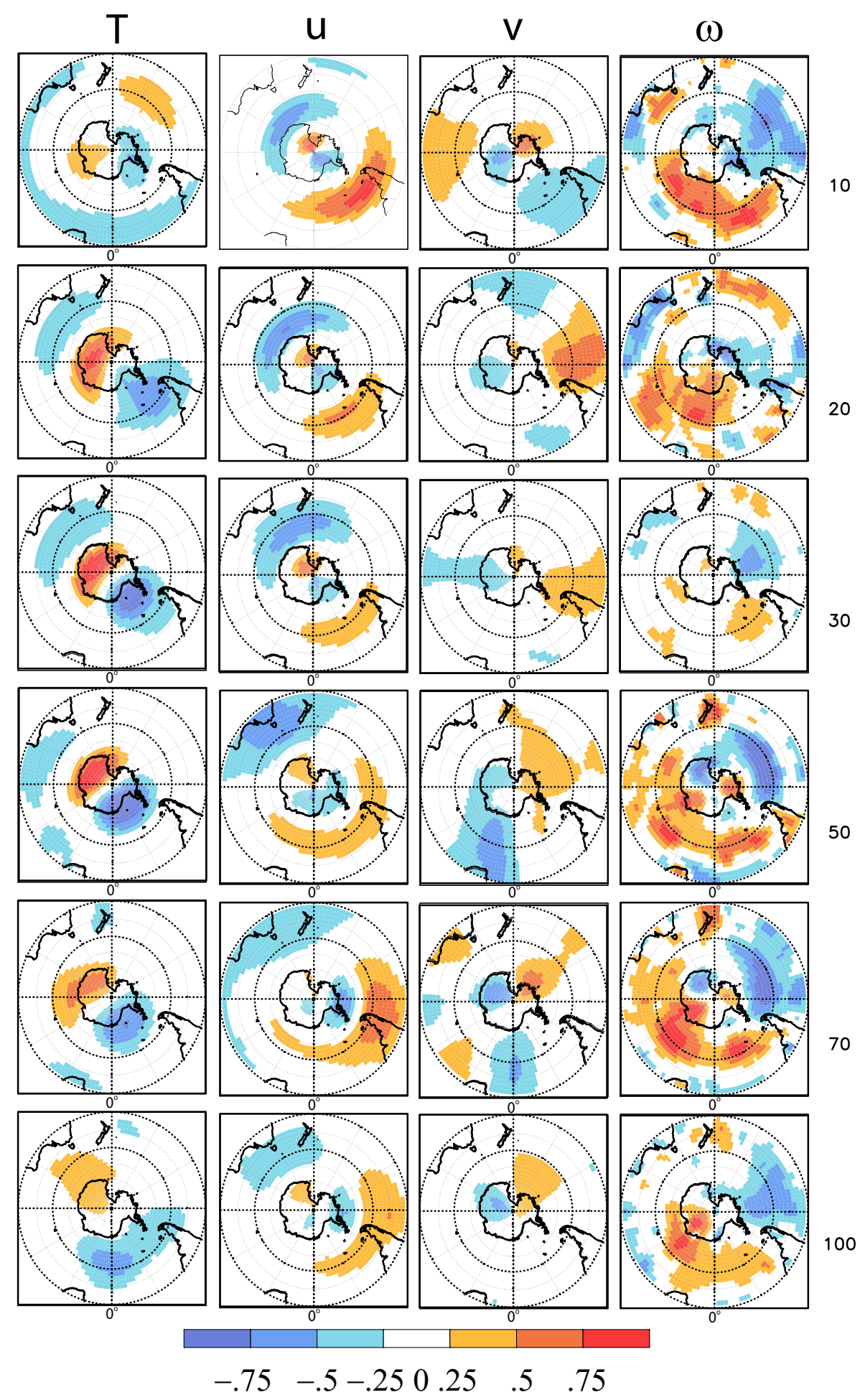

Fig. 8. Right heterogeneous correlations $\boldsymbol{r}_{Z_{21}}$ corresponding to the October GMCA2 right vectors at the pressure levels shown on the right (hPa). The inner and outer concentric circles are $50^{\circ} \mathrm{S}$ and $30^{\circ} \mathrm{S}$. 

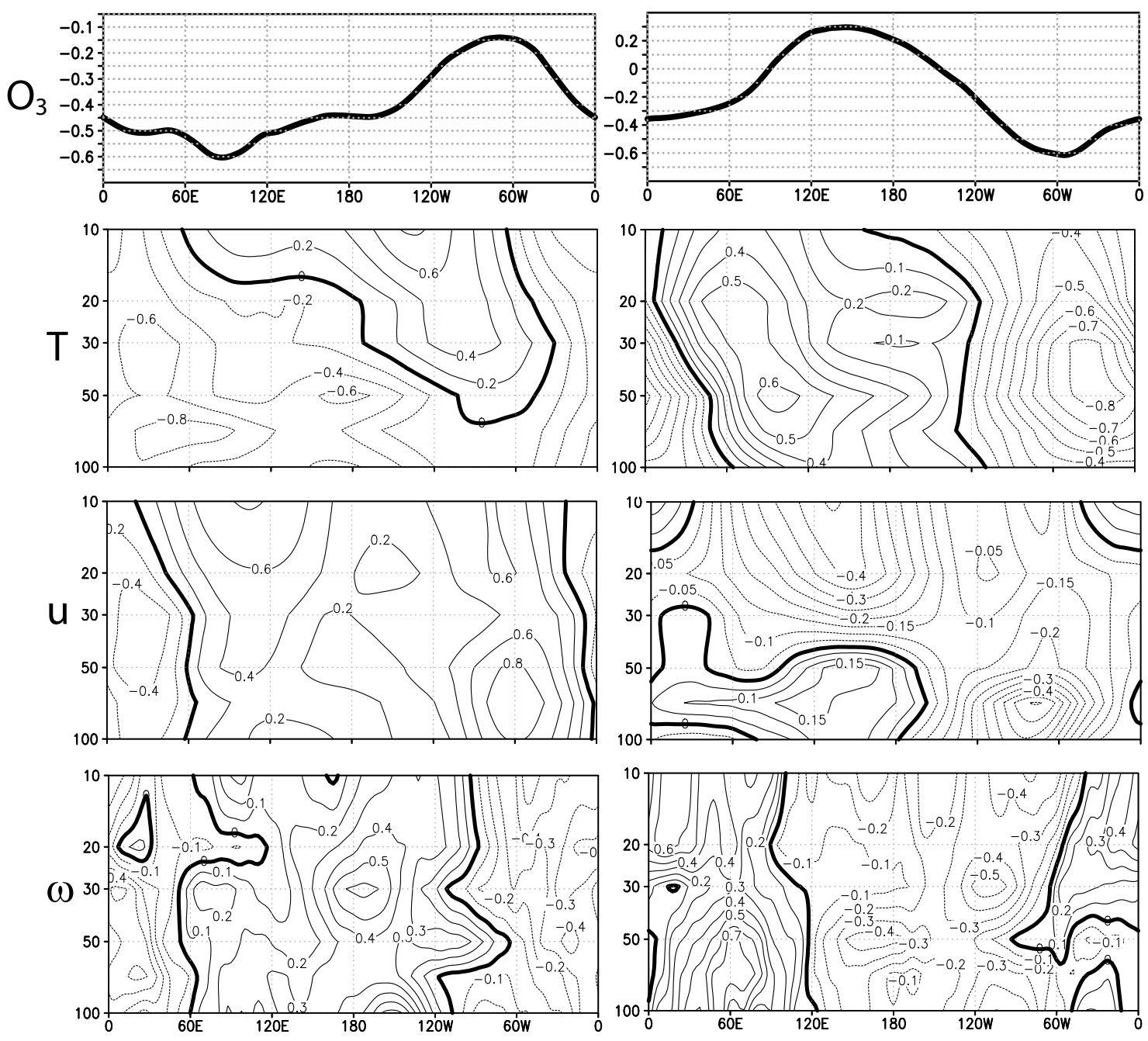

Fig. 9. Longitude/height cross sections for $S_{1}$ (left) and $S_{2}$ (right) and the October $Z_{1}$ (left) and $Z_{2}$ (right) heterogeneous correlations averaged between $50^{\circ}$ and $65^{\circ} \mathrm{S}$. Vertical scales are in $\mathrm{hPa}$.

patterns are weaker and more chaotic. Given the GMCA 1 is quasi-symmetric it is meaningful to look at its zonal means. Figure 7 shows the latitude height zonal means for $\mathbf{r}_{Z_{1}}$ for September, October and November. Consistent with Fig. 6 the averaged heterogeneous correlations show a shrinking of the polar vortex in terms of both $T$ and $u$ at all levels as time moves from September to November, that is passing through the time of the largest ozone hole. Figures 4-7 describe a distinct quasi-symmetric mode of variations in the Antarctic spring.

In contrast to $\mathbf{r}_{Z_{1}}$ the second most import GMCA $\mathbf{r}_{Z_{2}}$ patterns (Fig. 8) show much more asymmetry than the $\mathbf{r}_{Z_{1}}$ correlations, especially in temperature and zonal wind, near the Antarctic continent. The implied vertical motion, when $a_{2}(t)$ is positive, is generally upward in the Pacific and downward in the Indian Ocean sectors. The heterogeneous correlations for GMCA2 for the following month November (not shown) are generally weaker and more random. Thus, in contrast to the first GMCA the second describes an asymmetric mode for temperature and velocities at nearly all heights.

Figure 9 shows longitude/height cross sections for the zero lag $\mathbf{r}_{Z_{1}}$ and $\mathbf{r}_{Z_{2}}$ correlations averaged between $50^{\circ}$ and $65^{\circ} \mathrm{S}$. Figure 9 also includes the average $\mathbf{r}_{S_{1}}$ and $\mathbf{r}_{S_{2}}$ correlations in October for the same latitude. Focusing on the longitudes with the largest negative correlations for ozone (implying reduced ozone when $a_{1}(t)$ or $a_{2}(t)$ are positive), one sees that these longitudinal sectors generally correspond to the largest negative correlations in temperature. As expected from the thermal wind relation the positive meridional velocities tend to be in the quadrants of the positive to negative west-to-east temperature gradients. Furthermore, especially for $\mathbf{r}_{Z_{2}}$, there is a clear suggestion of vertical propagation with a westward tilt with increasing elevation of both temperature and meridional wind perturbations. Finally, the pressure velocity correlations tend to be positive (implying downward departures when $a_{i}(t)$ are positive) in the regions of negative meridional 


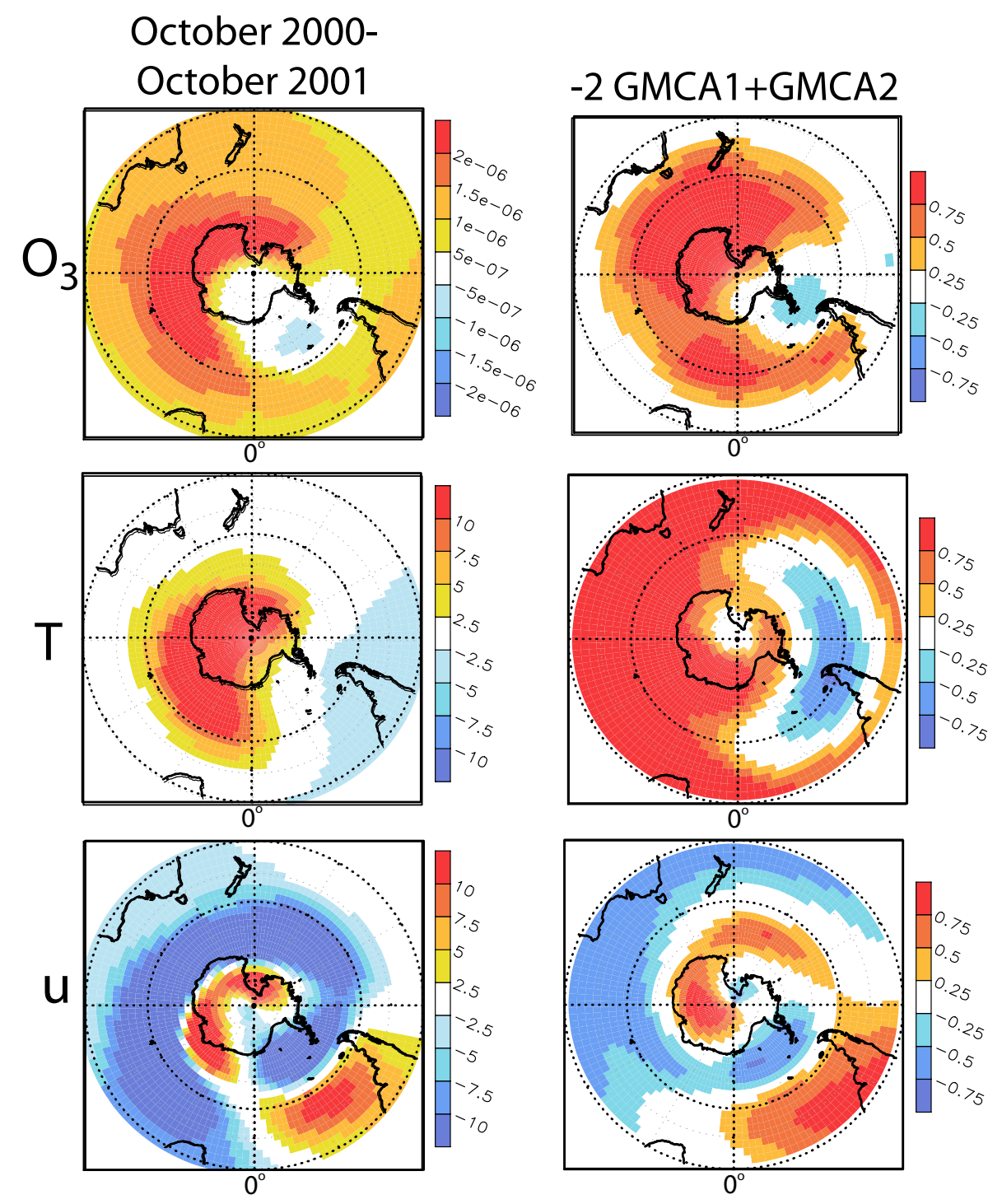

Fig. 10. October 2000 minus October $200150 \mathrm{hPa}$ differences. Right column: ERA-40 monthly means of zone mixing ratio (kg/kg), temperature $\left({ }^{\circ} \mathrm{C}\right)$ and zonal wind $(\mathrm{m} / \mathrm{s})$. Left column: heterogeneous correlation differences (see text and Eq. 6) for ozone mixing ratio, temperature and zonal wind. The inner and outer concentric circles are $50^{\circ} \mathrm{S}$ and $30^{\circ} \mathrm{S}$.

velocity correlation and vice versa. Thus transport towards (away) from the pole tends to be associated with downward (upward) motion at these longitudes. Finally, there is a general tendency for the largest magnitude correlations in $\mathbf{r}_{Z_{1}}$ to be at slightly higher elevations than those for $\mathbf{r}_{Z_{2}}$ as indicated by the approximate locations of the plus and minus signs in Fig. 9.

\section{Focus on the contrasts between October 2000 and 2001}

Based on the time series in Fig. 3 two of the years with the largest contrasting patterns of the quasi-symmetric and asymmetric modes are 2000 and 2001 . This is despite the fact that the concentrations of ozone destroying substances in the two years should be quite comparable, and that both years correspond to the negative phase of the QBO (see Fig. 3). The left side of Fig. 10 shows the $\mathrm{O}_{3}, T$ and $u$ differences for the $50 \mathrm{hPa}$ level for October 2000 (asymmetric) minus October 2001 (strongly symmetric) years. The patterns of the differences show an asymmetric pattern and have magnitudes of variations in the ozone mixing ratio, temperature and zonal wind differences in the range of $2 \mathrm{e}-6 \mathrm{~kg} / \mathrm{kg}, 10^{\circ} \mathrm{C}$ and $10 \mathrm{~m} / \mathrm{s}$, respectively. To compare with the GMCA results weighted means of the GMCA 1 and 2 results are used. Based on the time coefficients for these two years in Fig. 3 these means are given by 


$$
\begin{array}{ccc}
\text { October } 2000 & - & \text { October } 2001 \\
(-1 * \text { GMCA } 1+2 * \text { GMCA } & 2) & -(1 * \text { GMCA } 1+1 * \text { GMCA } 2)=(5) \\
-2 * \text { GMCA } 1 & + & \text { GMCA } 2
\end{array}
$$

The right side of Fig. 10 shows these weighted $50 \mathrm{hPa} \mathrm{O}_{3}, T$, and $u$ heterogeneous correlations. Especially in the region of the Antarctic continent the patterns of variation for the October 2000-2001 data differences and the weighted GMCAs are quite similar for all three fields. This is further confirmation of the importance of the symmetric and asymmetric modes. These results imply that October 2000 has much more ozone everywhere except near the Antarctic peninsular. Likewise the temperature is much higher over most of the region except in the South American sector. In addition the GMCA analysis shows positive correlations at lower latitudes. The zonal wind fields show strengthening over West Antarctica and in the South Atlantic and a weakening south of the Indian Ocean. These low latitude variations may be an indication of the sites of the sources of eddy wave energy leading to the stratospheric changes. The largest discrepancies between the left and right columns in Fig. 10 is in the two zonal wind analyses over the southern Pacific Ocean region.

In order to better understand the physical processes leading to differences in ozone mixing ratios in these two years the dynamical terms of the ozone budget have been calculated using 6-h ERA-40 data south of $30^{\circ} \mathrm{S}$. In spherical coordinates this budget (James, 1984) is given by

$$
\frac{\partial \mathrm{O}_{3}}{d t}=-\frac{u}{a \cos \theta} \frac{\partial \mathrm{O}_{3}}{d \lambda}-\frac{v}{a} \frac{\partial \mathrm{O}_{3}}{d \theta}-\omega \frac{\partial \mathrm{O}_{3}}{d p}+C
$$

where $a$ is the radius of the earth, $\theta$ and $\lambda$ are latitude and longitude, and $C$ mainly represents the chemical creation minus destruction of ozone. The comparable budget for potential temperature $\Theta$ (Bluestein, 1992) is given by

$$
\frac{\partial \Theta}{d t}=-\frac{u}{a \cos \theta} \frac{\partial \Theta}{d \lambda}-\frac{v}{a} \frac{\partial \Theta}{d \theta}-\omega \frac{\partial \Theta}{d p}+\frac{\Theta}{c_{p} T} Q
$$

where $Q$ is the radiative heating rate, and $c_{p}$ is the heat capacity at constant pressure. The transport terms of these equations (first three terms on the right hand side) are calculated in flux forms using centered finite differences. It should be noted that since the data used in these calculations are 6-h samples, these resolved transport terms include the influence of transient as well as standing eddies.

Figure 11 shows the differences between the values for October 2000 minus October 2001 for the sum of the first three terms on the right side of Eqs. (7) and (8). The potential temperature terms have been converted to temperature by using the Poisson Equation. The calculated $\frac{\partial O_{3}}{d t}$ and $\frac{\partial \Theta}{d t}$ rates are both relatively small (not shown). Both budgets illustrated in Fig. 11 show a strong wave number one pattern with $\mathrm{O}_{3}$ richening and heating over most of the Eastern
October 2000October 2001
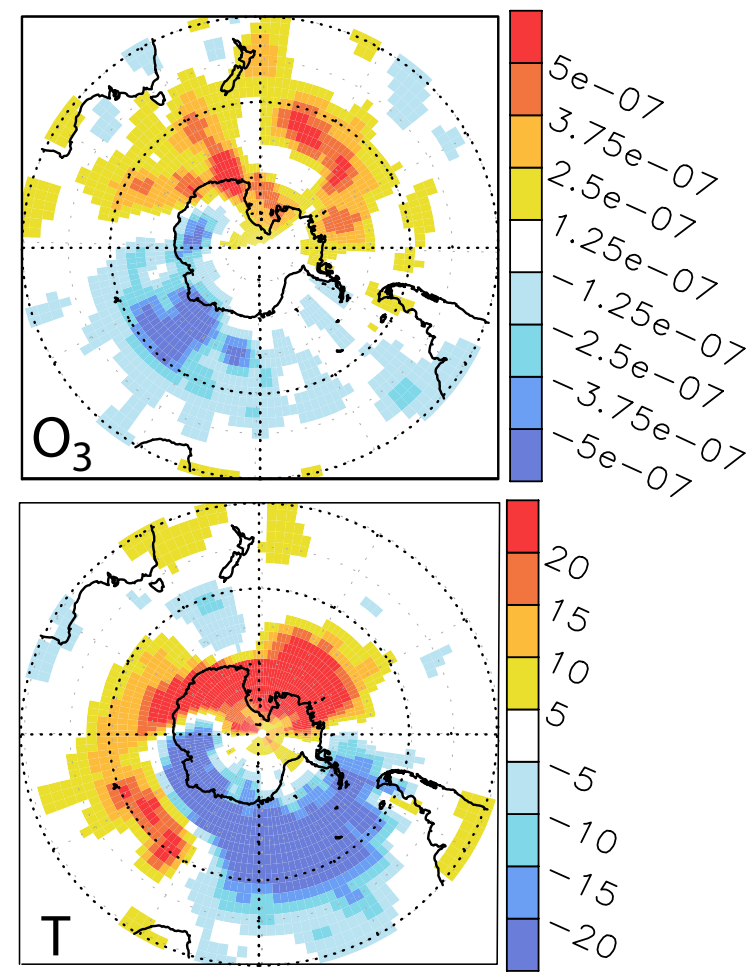

Fig. 11. October 2000 minus October $200150 \mathrm{hPa}$ differences. Top: First three terms of the right side of the ozone conservation equation (Eq. 8) $\left(\right.$ day $\left.^{-1}\right)$. Bottom: First three terms of the right side of the potential temperature conservation equation (Eq. 8) $\left(\mathrm{Kday}^{-1}\right)$. The inner and outer concentric circles are $50^{\circ} \mathrm{S}$ and $30^{\circ} \mathrm{S}$.

Hemisphere and freshening and cooling over a large part of the Western Hemisphere. The patterns and values for differences between averages of September and October for these two years are very similar (not shown). The primary region where the patterns in Fig. 11 differ is in the Indian Ocean (the eight o'clock sector on the maps). These maps of transport related changes may be compared to the observed differences in ozone mixing ratio and temperature for these two months shown in Fig. 10. Although some of the observed differences may be attributable to the dynamical processes illustrated in Fig. 11, two important discrepancies exist. First, the dynamical changes are dominated by wave number one and the resultant changes are much more axisymmetric. Second, the rates of changes in Fig. 11 are much larger than necessary to bring about the differences seen in Fig. 10. Thus, both radiative heating and cooling and ozone creation and destruction must be balancing the dynamical changes to a great extent (Brasseur and Solomon, 2007).

Although the patterns shown in Figs. 10 and 11 are not symmetric about the pole, it is still illustrative to look at the zonal means of the differences between October 2000 and 

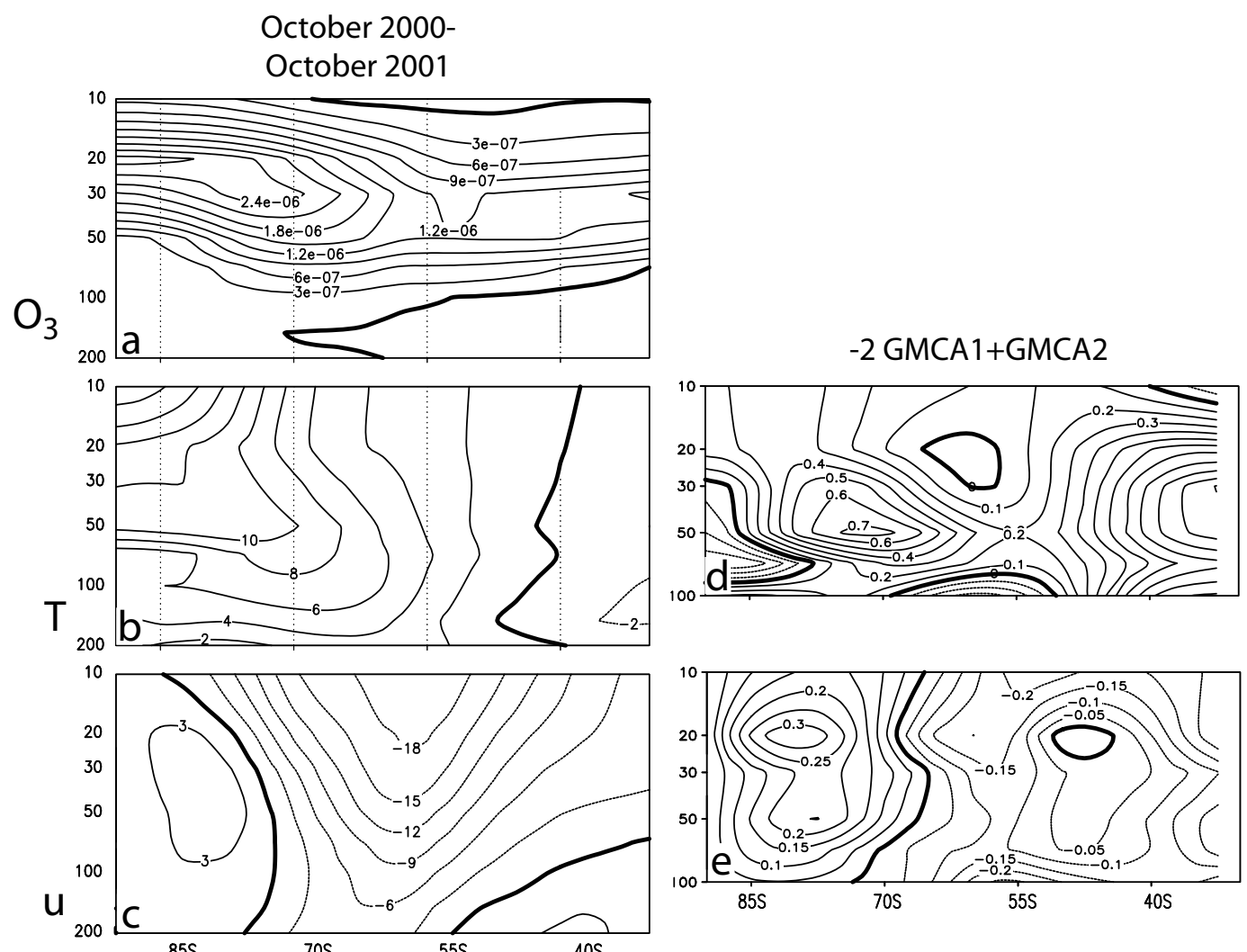

Fig. 12. October 2000 minus October 2001 differences of zonal means. ERA-40 monthly mean ozone (a), temperature, and (c) zonal wind. Heterogeneous correlation differences (see text and Eq. 6) for temperature (d) and zonal wind (e). Vertical scales are in hPa.
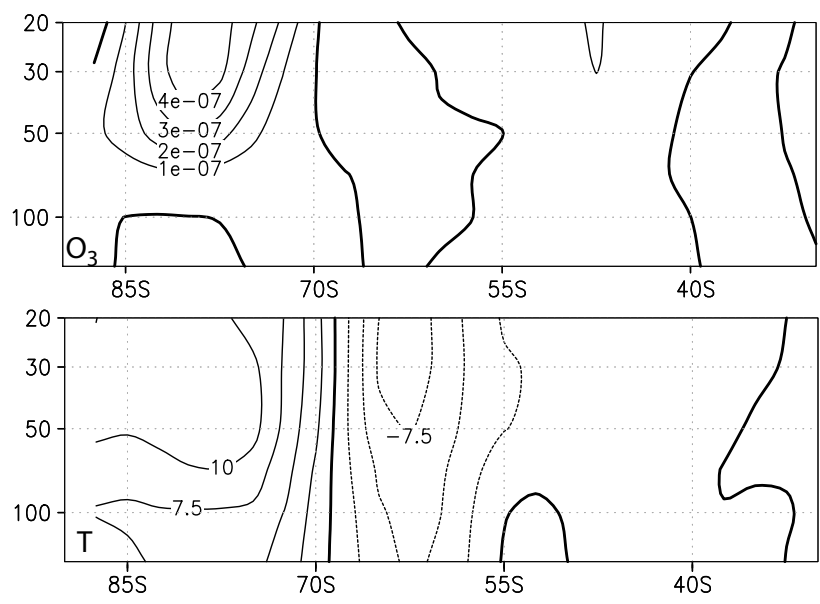

Fig. 13. Zonal means of the 2000-2001 October differences. Top: Ozone (sum of first three terms on the right side of Eq. (6); day ${ }^{-1}$ ). Bottom: Temperature (sum of first three terms on the right side of Eq. 7; $\mathrm{Kday}^{-1}$ ). Vertical scales are in $\mathrm{hPa}$.

2001. Figure 12 shows latitude/height plots of the zonal means of the differences between October 2000 and 2001 for the $\mathrm{O}_{3}, T$ and $u$ from the ERA-40 reanalysis output. South of about $60^{\circ} \mathrm{S}$ these plots show that ozone and temperature are greater in 2000 at nearly all levels. Furthermore, for October 2000 minus 2001 the zonal winds at all levels are stronger near the pole and much weaker near $60^{\circ} \mathrm{S}$. The ozone and zonal wind results may be compared to the zonal means of the weighted average (as in Fig. 10; Eq. 6) heterogeneous correlations from GMCA 1 and 2. In general agreement with the observational differences, there are positive temperature correlations near $75^{\circ} \mathrm{S}$ at all levels. Furthermore, the pattern of weighted heterogeneous correlations for zonal wind are positive near the pole and negative near $60^{\circ} \mathrm{S}$ at all levels, which is also in general agreement with the observations. Thus, despite the fact that the observational analysis is based on differences between two single years and the GMCA patterns are for only the first two modes of co-variability quite remarkable similarities exist. The broad agreement between the difference patterns in the basic data and the weighted heterogeneous correlations again helps to give confidence to the assertion that GMCA 1 and 2 represent true modes of variation of the Antarctic stratosphere.

Figure 13 shows the 2000 minus 2001 October zonal mean differences for the budget terms (Eqs. 7 and 8) for the ozone and temperature for the levels between 20 and $150 \mathrm{hPa}$. This figure shows that there is a tendency for ozone mixing ratio 
to increase south of $70^{\circ} \mathrm{S}$ at levels above $100 \mathrm{hPa}$ with minimal changes elsewhere. Thus, south of about $70^{\circ} \mathrm{S}$ the transport differences are contributing directly to the observed positive differences in both temperature and ozone mixing ratio seen in Fig. 12. Equatorward, differences in chemical production or destruction and radiative heating or cooling are necessary to create the observed differences in ozone mixing ratio and temperature. In nearly all regions, especially equatorward of about $70^{\circ} \mathrm{S}$, the changes in the dynamically driven production rates of ozone mixing ratio and temperature are much larger than the observed perturbations. This implies again that both chemical and radiative processes are important nearly everywhere.

\section{Summary and discussion}

Generalized Maximum Covariance Analysis (GMCA) has been applied to diagnosing the temperature, wind and pressure velocity variations related to October perturbations in Southern Hemisphere ozone associated with the Antarctic ozone hole. This analysis identifies the most important patterns of co-variability between interannual $50 \mathrm{hPa}$ ozone mixing ratio variations associated with the October ozone hole and temperature, zonal, meridional and vertical velocities between 100 and $10 \mathrm{hPa}$ in the same region at lags and leads out to two months. The first two sets of GMCA time coefficients have variations showing moderate trends and larger year-to-year variability. The associated heterogeneous correlations of both ozone variations and the associated departures in temperature and winds may be characterized as quasi-symmetric and asymmetric modes of variation about the pole.

The robustness of the GMCA results has been confirmed by recalculation of the GMCAs using slightly different $\mathbf{S}$ and $\mathbf{Z}$ data sets. For instance instead of $\mathbf{S}$ being represented by the first ten PCs of 50hPa ozone mixing ratio a second analysis used the PC's associated with an EOF analysis for all layers between 10 and $100 \mathrm{hPa}$. In another analysis the original $\mathbf{S}$ field was related to the $T, u, v$, and $\omega$ departures only at zero lag, that is October of each year. In a third analysis the $\mathbf{Z}$ data was for the levels between 20 and $200 \mathrm{hPa}$. In all three of these cases the two most GMCA time coefficients (corresponding to Fig. 3) and zero-lag patterns (corresponding to Figs. 5 and 8 ) are very similar to those illustrated (not shown). Thus, these results are not sensitive to small changes in either the $\mathbf{S}$ or $\mathbf{Z}$ fields. On the other hand the dominant GMCAs were found to be different from those illustrated if the number of lags was expanded from \pm 2 months to \pm 11 months. This is apparently related to the fact that the variations in months not near the Antarctic spring are poorly related to the ozone hole.

The differences between the years 2000 and 2001 were used to better understand the symmetric and asymmetric modes. The horizontal structure of the $50 \mathrm{hPa}$ differences in ozone mixing ratios, temperature and zonal winds of the ERA-40 values and the appropriate averages of the dominant GMCAs are quite similar. In particular both analyses show that positive ozone and temperature departures over West Antarctica and the nearby oceans are associated with negative departures near South America. These ozone and temperature patterns, which have magnitudes of approximately $2 \mathrm{e}-6 \mathrm{~kg} / \mathrm{kg}$ and $10^{\circ} \mathrm{C}$, are accompanied by increased zonal winds of up to about $10 \mathrm{~m} / \mathrm{s}$ over much of Antarctica and near South America and decreases over broad regions elsewhere. These changes are associated with strong wave number one patterns in the transport terms of both ozone mixing ratio and temperature such that increases in both are seen the Eastern Hemisphere, and the transition from positive to negative departures occurs near South America.

These results suggest a particular importance to the South American sector, and perhaps to perturbations in the flow associated with the Andes. This is in general agreement with Peters et al. (2007), who find for the large 2002 Antarctic spring warming that there is a strong excitation of Rossby waves in the region of the Waddell Sea. Peters et al. also support the assertion that topographic effects are important to wave activity in the region. On the other hand Nishii and Nakamura (2004) stress that this same 2002 spring warming is related to a blocking pattern over the middle latitudes of the South Atlantic. However, neither of these authors indicates the strong role for radiation and chemistry in the spring warming indicated by the current budget analyses.

Overall, these results suggest strong year to year variations of distinct quasi-symmetric and anti-symmetric modes of co-variability. The associated time coefficients of both of these modes not only have clear long-term trends, but also have prominent year-to-year variability. This latter variability might strongly influence the interpretation of studies which rely upon looking at differences from a small number of arbitrarily chosen years, such as Canziani et al. (2008).

Some of these results may be summarized by a simple heuristic model starting with changes in ozone mixing ratio over the pole induced by ozone destroying substances associated with polar stratospheric clouds in the Antarctic spring as is illustrated in the following expression:

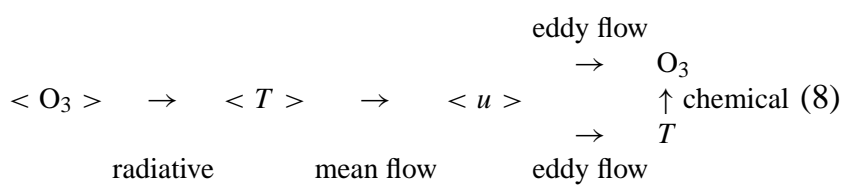

where $<>$ identifies a zonal mean. The first three terms in this expression are assumed to be largely zonally symmetric in which for instance a lowered ozone mixing ratio decreases the temperature near the pole, which in turn is associated with a strengthening of the polar vortex. The latter terms are related to the eddy responses associated with these zonal wind changes. For instance, an interaction with the Andes in the southern most part of South America may induce a wave 
number one response in the flow which creates asymmetric patterns of ozone and temperature departures. The temperature changes in turn induce chemically moderated changes in ozone mixing ratio. This set of processes gives rise to the quasi-symmetric and asymmetric modes of temperature and ozone variations seen in the observations. Alternately, tropospheric eddy wave forcing could induce both symmetric and asymmetric variations, which are modified by both radiation and chemistry. These ideas stress the importance of not only dynamics, but also chemistry and radiation, in the year-to-year variations in the Antarctic ozone hole.

The basic conclusions, summarized in this paper, could be further studied in a number of ways. One useful task would to more thoroughly explore the variations associated with GMCAs 3 and 4. Although the time variations of these functions are uncorrelated with those discussed, understanding these modes may be important in defining the full range of variability in this region and choosing years to be considered for case studies. Other additional studies include repeating these analyses with the NCEP/NCAR reanalysis (Kistler et al., 2001) or by combining reanalysis winds with satellite derived ozone and temperature. Furthermore, the case study methodology could be applied to other years, which had documented asymmetric flows. Additional diagnostics studies could utilize the methods of Peter et al. (2007) to explore the differences in middle latitude wave driving, which are responsible for the two modes identified here.

Acknowledgements. ERA-40 data were provided by the European Centre for Medium-Range Weather Forecasting from their web site at http://data.ecmwf.int/data/d/era40_daily/. This work was partially supported by NSF grant ATM-0733698.

Edited by: M. Dameris

\section{References}

Brasseur, G. P. and Solomon, S.: Aeronomy of the middle atmosphere : chemistry and physics of the stratosphere and mesosphere, Springer, Dordrecht, Germany, 2007.

Bretherton, C. S., Smith, C., and Wallace, J. M.: An intercomparison of methods for finding coupled patterns in climate data, J. Climate, 5, 541-560, 1992.

Bluestein, H. B.: Synoptic-Dynamic Meteorology in Midlatitudes, volume 1, Oxford University Press, New York, 1992.

Canziani, P. O., Malanca, F. E., and Agosta, E. A.: Ozone and upper troposphere/lower stratosphere variability and change at southern midlatitudes 1980-2000: Decadal variations, J. Geophys. Res., 113, D20101, doi:10.1029/2007JD009303, 2008.
Dethof, A. and Hólm, E. V.: Ozone assimilation in ERA-40, Q. J. Roy. Meteorol. Soc., 124, 1783-1808, 2004.

Grytsai, A. V., Evtushevsky, O. M., and Milinevsky, G. P.: Anomalous quasi-stationary planetary waves over the Antarctic region in 1988 and 2002, Ann. Geophys., 26, 1101-1108, 2008, http://www.ann-geophys.net/26/1101/2008/.

James, I. N.: Introduction to Circulating Atmospheres, Cambridge University Press, Cambridge, 1994.

Kistler, R., Kalnay, E., Collins, W., Saha, S., White, G., Woollen, J., Chelliah, M., Ebisuzaki, W., Kanamitsu, M., Kousky, V., van den Dool, H., Jenne, R., and Fiorino, M.: The NCEP-NCAR 50year reanalysis: Monthly means CD-ROM and documentation, B. Am. Meteorol. Soc., 82, 247-267, 2001.

Le Sommer, J., Teitelbaum, H., and Zeitlin, V.: Global estimates of equatorial inertia-gravity wave activity in the stratosphere inferred from ERA40 reanalysis, Geophys. Res. Lett., 33, L07810, doi:10.1029/2005GL024873, 2006.

Nishii, K. and Nakamura, H.: Tropospheric influence on the diminished Antarctic ozone hole in September 2002, Geophys. Res. Lett., 31, L16103, doi:10.1029/2004GL019532, 2004.

Pascoe, C. L., Gray, L. J., Crooks, S. A., Juckes, M. N., and Baldwin, M. P.: The quasi-biennial oscillation: Analysis using ERA-40 data, J. Geophys. Res., 110, D08105, doi:10.1029/2004JD004941, 2005.

Peters, D., Vargin, P., and Körnich, H.: A study of the zonally asymmetric tropospheric forcing of the austral vortex splitting during September 2002, Tellus, 59A, 384-394, 2007.

Randel, W., Udelhofen, P., Fleming, E., Geller, M., Gelman, M., Hamilton, K., Karoly, D., Ortland, D., Pawson, S., Swinbank, R., Wu, F., Baldwin, M., Chanin, M. L., Keckhut, P., Labitzke, K., Remsberg, E., Simmons, A., and Wu, D.: The SPARC intercomparison of middle-atmosphere climatologies, J. Climate, 17, 986-1003, 2004.

Rowland, F. S.: Stratospheric ozone depletion, Phil. Trans. R. Soc., 361, 769-790, 2006.

Uppala, S. M., Kallberg, P. W., Simmons, A. J., Andrae, U., Bechtold, V. D., Fiorino, M., Gibson, J. K., Haseler, J., Hernandez, A., Kelly, G. A., Li, X., Onogi, K., Saarinen, S., Sokka, N., Allan, R. P., Andersson, E., Arpe, K., Balmaseda, M. A., Beljaars, A. C. M., Van De Berg, L., Bidlot, J., Bormann, N., Caires, S., Chevallier, F., Dethof, A., Dragosavac, M., Fisher, M., Fuentes, M., Hagemann, S., Holm, E., Hoskins, B. J., Isaksen, L., Janssen, P. A. E. M., Jenne, R., McNally, A. P., Mahfouf, J. F., Morcrette, J. J., Rayner, N. A., Saunders, R. W., Simon, P., Sterl, A., Trenberth, K. E., Untch, A., Vasiljevic, D., Viterbo, P., and Woollen, J.: The ERA-40 re-analysis, Q. J. Roy. Meteorol. Soc., 131, 2961-3012, 2005.

Von Storch, H. and Zwiers, F. W.: Statistical Analysis in Climate Research, Cambridge University Press, Cambridge, 1999.

Weare, B. C.: Multi-lag Singular Value Decomposition Analysis of ENSO Convection with Tropical Stratospheric Temperature, J. Climate, 21, 6556-6568, 2008.

Weare, B. C.: Structure of stratospheric wave responses to ENSO convection, J. Climate, in press, 2009. 